\title{
Factors associated with hospital and intensive care admission in paediatric SARS-CoV-2 infection: a prospective nationwide observational cohort study
}

\author{
Anita Uka ${ }^{1,2} \cdot$ Michael Buettcher $^{3} \cdot$ Sara Bernhard-Stirnemann ${ }^{4} \cdot$ Yves Fougère $^{5} \cdot$ Dehlia Moussaoui $^{6}$. \\ Lisa Kottanattu ${ }^{7} \cdot$ Noémie Wagner $^{6} \cdot$ Petra Zimmermann ${ }^{1,2,8} \cdot$ Nicole Ritz $^{9,10,11}$ (D) . \\ Swiss Paediatric Surveillance Unit (SPSU)
}

Received: 17 May 2021 / Revised: 27 July 2021 / Accepted: 26 September 2021 / Published online: 29 November 2021

(c) The Author(s) 2021

\begin{abstract}
Coronavirus disease 2019 (COVID-19) is usually less severe in children compared to adults. This study describes detailed clinical characteristics, treatment and outcomes of children with COVID-19 in a non-hospitalised and hospitalised setting and quantifies factors associated with admission to hospital and intensive care unit in children with SARS-CoV-2 infection on a nationwide level. Data were collected through the Swiss Paediatric Surveillance Unit from children $<18$ years with confirmed SARS-CoV-2 infection. All 33 paediatric hospitals in Switzerland reported non-hospitalised and hospitalised cases from March 1 to October 31, 2020 during both pandemic peaks. In total, 678 children were included. The median age was 12.2 years (IQR 5.0-14.6), 316 (46.6\%) were female and 106 (15.6\%) had comorbidities. Overall, 126 (18.6\%) children were hospitalised of whom $16(12.7 \%)$ required ICU admission. Comorbidities were the only factor associated with hospital admission in a multivariable regression analysis (odds ratio $3.23,95 \% \mathrm{CI} 1.89$ to 5.50 ; $p$-value $<0.01$ ). Children with preexisting comorbidities did not require ICU admission more often. Hospitalised children more often presented with fever (96 [76.2\%] vs 209 [38.1\%], $p$-value $<0.01)$ and rash $(16$ [12.8\%] vs $6[1.1 \%]$, $p$-value $<0.01)$. Anosmia/dysgeusia was more prevalent in non-hospitalised children (73 [13.3\%] vs 3 [2.4\%], $p$-value $<0.01)$. In hospitalised children, oxygen treatment was required in 34 (27.0\%), inotropes in nine (7.3\%) and mechanical ventilation in eight (6.3\%) cases. Complications were reported in 28 (4.1\%) children with cardiovascular complications being most frequent (12 [1.8\%]). Three deaths were recorded.

Conclusion: This study confirms that COVID-19 is mostly a mild disease in children. Fever, rash and comorbidities are associated with higher admission rates. Continuous observation is necessary to further understand paediatric COVID-19, guide therapy and evaluate the necessity for vaccination in children.
\end{abstract}

\section{What is Known:}

- Clinical manifestations of SARS-CoV-2 infection in children vary from asymptomatic to critical disease requiring intensive care unit admission.

- Most studies are based on hospitalised children only; currently, there is limited data on non-hospitalised children.

What is New:

- The clinical spectrum and severity of COVID-19 is influenced by age: in children less than 2 years, fever, cough and rhinorrhoea are the most common symptoms and in adolescents, fever, cough and headache are more common.

- Hospitalised children more often presented with fever and rash, while anosmia/dysgeusia is more prevalent in non-hospitalised children.

- Children with pre-existing comorbidities are more frequently hospitalised but do not require ICU admission more often.

Keywords COVID-19 $\cdot$ Child $\cdot$ Epidemiology $\cdot$ Clinical presentation $\cdot$ Outcome $\cdot$ Transmission

Petra Zimmermann and Nicole Ritz have shared authorship.

Nicole Ritz

Nicole.ritz@unibas.ch

\section{Abbreviations}

ACE-2 Angiotensin-converting enzyme 2

COVID-19 Coronavirus disease-2019

EBV Epstein-Barr Virus

ICU Intensive care unit

Extended author information available on the last page of the article 


\section{IQR Interquartile range \\ IVIG Intravenous immunoglobulins \\ MIS-C Multisystem inflammatory syndrome in children \\ PIMS-TS Paediatric inflammatory multisystem syndrome-temporally associated with SARS-CoV-2 \\ PCR Polymerase chain reaction}

SARS-CoV-2 Severe acute respiratory syndrome coronavirus 2

SPSU Swiss Paediatric Surveillance Unit

\section{Introduction}

Compared to adults, coronavirus disease 2019 (COVID19) manifests differently in infants, children and adolescents [1-5]. Although, the disease severity is often milder in children, paediatric patients may also develop severe disease requiring admission to intensive care unit (ICU) and may very rarely die from COVID-19 [6-8]. Additionally, children presenting with a delayed inflammatory disease called 'paediatric inflammatory multisystem syndrome-temporally associated with SARS-CoV-2 (PIMS-TS)' or'multisystem inflammatory syndrome in children (MIS-C)' have been reported [7, 9-15].

To date, the data on SARS-CoV-2 infection in children and adolescents either come from non-hospitalised children with limited clinical information or from hospitalised children only. This limits the knowledge in paediatric COVID19 on factors associated with admission including clinical presentation and risk factors such as age, sex or comorbidities. This study presents epidemiological data from active surveillance of SARS-CoV-2 infections in non-hospitalised and hospitalised children in Switzerland and provides insights regarding risk factors for admission.

\section{Methods}

\section{Study design and population}

Our study is a prospective nationwide observational cohort study that describes detailed clinical characteristics and outcomes of children with laboratory-confirmed COVID-19 in a non-hospitalised and hospitalised setting. Paediatric SARSCoV-2 infections are actively monitored in an observational study by the Swiss Paediatric Surveillance Unit (SPSU, http://www.spsu.ch) since March 2020. The current analysis includes data from March 1 to October 31, 2020. All 33 paediatric and neonatological hospitals in Switzerland participated and notify cases monthly. Upon notification, the investigators sent the reporting centres an electronic clinical report form through RedCap or in paper form (see supplementary data Questionnaire) [16]. All data were recorded anonymously and reviewed by the investigators and further clarified with the reporting physician when needed. The study has received ethical approval by the Ethikkommission Nordwest- und Zentralschweiz (EKNZ 2020-01,130).

\section{Case definition}

Children and adolescents $<18$ years of age who presented to a Swiss paediatric hospital and received ambulatory or hospitalised care were included if diagnosed with COVID-19 by detection of SARS-CoV-2 from a clinical specimen using a validated polymerase chain reaction (PCR) test or serology. Retrospective screening of patients with the following criteria was used for identification of potential PIMS-TS cases: PIMS-TS reported by the clinician, SARS-CoV-2 serology performed, ICU admission or cardiac changes.

\section{Statistical analysis}

Continuous data were summarised using median and interquartile ranges. Categorical data were presented as percentage. Categorical data were compared using the $\chi^{2}$ test, with $p$-values $<0.05$ considered as significant. Cooccurrence symptoms were clustered using the K-means clustering solution and visualised with a heat map. A multivariable logistic regression model of risk of admission was fitted by including all variables used for univariable analysis origin. For the assessment of age, the following groups were made 0 to $<2,2$ to $<5,5$ to $<10$ and 10 to $<18$ years of age. $\mathrm{R}$ (Version 1.2.5019) was used for statistical analyses.

\section{Results}

\section{Study population}

A detailed dataset was returned for 682 cases, of which 678 were included in the final analysis. Reasons for exclusion were duplication in reporting $(n=3)$ and age $\geq 18$ years $(n=1)$. The age of the children ranged from 7 days to 17.9 years with a median of 12.2 years (interquartile range (IQR) 5.0-14.6) (Table 1). Most of the children were Caucasian (532 [78.5\%]), followed by Arabic (29 [4.3\%]), Hispanic (27 [4.0\%]), Black (18 [2.7\%]) and Asian (10 [1.5\%]). Ethnicity was unknown for $62(9.1 \%)$ children. Numbers of reported children over time are shown in Fig. 1 and stratified to age group in supplementary data 
Fig. S1. Geographical and temporal distribution of SARSCoV-2 cases in Swiss cantons (political states) is shown in supplementary data Fig. S2.

\section{Hospitalisation and management}

Overall, 126 (18.6\%) children were hospitalised of which 14 $(11.1 \%)$ were hospitalised for other reasons than infection with SARS-CoV-2. A total of $16(12.7 \%)$ children required ICU admission for the following reasons: hemodynamic instability $(n=8)$, respiratory failure $(n=4)$, prematurity $(n=1)$, coma $(n=1)$, cardiovascular arrest $(n=1)$, neurogenic shock $(n=1)$. One adolescent with a mild upper respiratory tract illness was admitted to ICU for a nonCOVID-19-related reason (neurogenic shock after an accident). Ethnicity of the children admitted to ICU were Caucasian $(n=8)$, Black $(n=4)$, Hispanic $(n=3)$ and unknown $(n=1)$. Oxygen was required in $34(27.0 \%)$, inotropes in nine $(7.3 \%)$ and mechanical ventilation in eight $(6.3 \%)$ hospitalised cases. Complications were reported in 25 (19.8\%) hospitalised children with cardiovascular complications being most frequent (11 [8.7\%]). A total of 48 children were retrospectively analysed for potential PIMS-TS of which 17 children were identified as cases based on available data (eight non-ICU admitted, nine ICU admitted). Three deaths were recorded.

Overall, most children (646 [95.3\%]) did not receive medication. Specific treatment was given to $10(1.8 \%)$ of non-hospitalised, 15 (13.6\%) of hospitalised and 12 (75.0\%) of ICU-admitted children. Among hospitalised children (non-ICU admitted), six (5.5\%) received corticosteroids, two (1.8\%) each hydroxychloroquine and intravenous immunoglobulins (IVIG) and one $(0.9 \%)$ tocilizumab. Among ICU-admitted children, nine (56.3\%) received biologicals (anakinra $(n=7)$, tocilizumab $(n=2)$ ), seven $(43.8 \%)$ each corticosteroids, IVIG and two (12.5\%) hydroxychloroquine. A combination of IVIG and corticosteroids was given to five children with suspected PIMS-TS in the ICU group. No further treatment including remdesivir was given (supplementary data Questionnaire). The median duration of hospitalisation for non-ICU admitted children was 3.0 days (IQR 2.0-4.0) and for children admitted to ICU 14 (IQR 4.75-15.25) days (supplementary data Fig. S3).
Table 1 Baseline characteristics and clinical information of children with SARS-CoV-2 infection

\begin{tabular}{|c|c|c|c|c|}
\hline & $\begin{array}{l}\text { Overall } \\
n(\%) \\
n=678\end{array}$ & $\begin{array}{l}\text { Non-hospitalised } \\
n(\%) \\
n=552\end{array}$ & $\begin{array}{l}\text { All hospitalised* } \\
n(\%) \\
n=126\end{array}$ & $\begin{array}{l}\text { ICU } \\
n(\%) \\
n=16\end{array}$ \\
\hline \multicolumn{5}{|l|}{ Age in years } \\
\hline$<2$ & $117(17.3)$ & $52(9.4)$ & $65(51.6)$ & $4(25.0)$ \\
\hline 2 to $<5$ & $49(7.2)$ & $42(7.6)$ & $7(5.6)$ & $0(0.0)$ \\
\hline 5 to $<10$ & $99(14.6)$ & $85(15.4)$ & $14(11.1)$ & $2(12.5)$ \\
\hline$\geq 10$ & $413(60.9)$ & $373(67.6)$ & $40(31.7)$ & $10(62.5)$ \\
\hline Age $<1$ month & $17(2.5)$ & $5(0.9)$ & $12(9.5)$ & $1(6.2)$ \\
\hline Female & $316(46.6)$ & $262(47.5)$ & $54(42.9)$ & $5(31.3)$ \\
\hline Comorbidities & $106(15.6)$ & $72(13.0)$ & $34(27.0)$ & $5(31.3)$ \\
\hline \multicolumn{5}{|l|}{ Symptoms } \\
\hline Fever & $305(45.3)$ & $209(38.1)$ & $96(76.2)$ & $11(68.8)$ \\
\hline Cough & $277(41.2)$ & $229(41.8)$ & $48(38.4)$ & $4(26.7)$ \\
\hline Rhinorrhoea & $191(28.4)$ & $142(25.9)$ & $49(39.2)$ & $4(26.7)$ \\
\hline Pharyngitis & $187(27.8)$ & $164(29.9)$ & $23(18.4)$ & $3(20.0)$ \\
\hline Anosmia/dysgeusia & $76(11.3)$ & $73(13.3)$ & $3(2.4)$ & $1(6.7)$ \\
\hline Abdominal pain & $76(11.3)$ & $60(10.9)$ & $16(12.8)$ & $5(33.3)$ \\
\hline Diarrhoea & $68(10.1)$ & $46(8.4)$ & $22(17.6)$ & $5(33.3)$ \\
\hline Vomiting & $59(8.8)$ & $37(6.8)$ & $22(17.6)$ & $5(33.3)$ \\
\hline Respiratory distress & $49(7.3)$ & $18(3.3)$ & $31(24.6)$ & $10(62.5)$ \\
\hline Rash & $22(3.3)$ & $6(1.1)$ & $16(12.8)$ & $5(33.3)$ \\
\hline Oxygen saturation $<92 \%$ & $18(2.7)$ & $1(0.2)$ & $17(13.5)$ & $6(37.5)$ \\
\hline Asymptomatic & $39(5.8)$ & $35(6.3)$ & $4(3.2)$ & $0(0.0)$ \\
\hline
\end{tabular}

$I C U$ intensive care unit

*Numbers of hospitalised children include those admitted to ICU 
Fig. 1 Number of children with SARS-CoV-2 infection over time

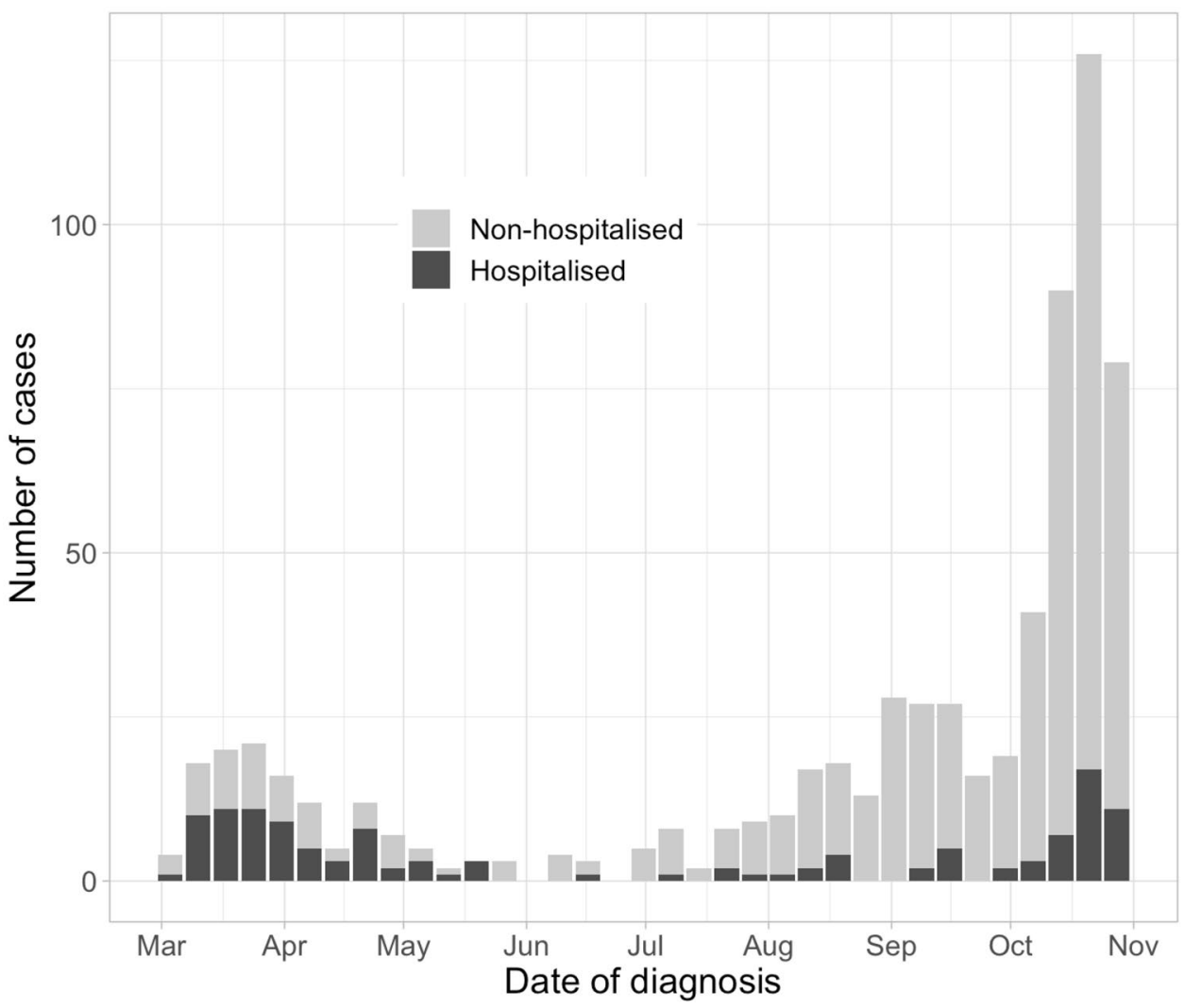

\section{Comorbidities}

A total of 106 (15.6\%) children had pre-existing medical conditions, the most common comorbidities beeing: respiratory (45 [42.5\%]), endocrinology (15 [14.2\%]), haematooncology (12 [11.3\%]) and cardiovascular (10 [9.4\%]) (details are listed in Table 2). Hospitalised children had significantly more comorbidities than non-hospitalised children ( $p$-value $<0.01)$. Five $(31.3 \%)$ children admitted to ICU had pre-existing comorbidities: three children had asthma/ bronchitis, a neonate had apnoea of prematurity (born at 29 weeks gestational age) and a 2-month-old infant had isolated microcephaly with a normal cerebral ultrasound. Children requiring admission to ICU did not have pre-existing medical conditions more frequently compared to non-ICUhospitalised children. A univariable regression analysis showed that children $\geq 2$ years of age were less likely to be hospitalised, and comorbidities increased the risk of hospitalisation in both uni- and multivariable regression analysis (Table 3).

\section{Symptoms}

Overall, fever was the most frequent symptom observed among children with COVID-19 (305 [45.3\%]) (Table 1). In children aged less than 2 years of age, fever, cough and rhinorrhoea were the most common symptoms, and in adolescents between 10 and 18 years of age, fever, cough and headache were more commonly reported (a detailed distribution of symptoms according to age group is presented in Fig. 2). Fever and rash were more common in hospitalised compared with non-hospitalised children ((96 [76.2\%] vs 209 [38.1\%], $p$-value $<0.001)$ and $(16$ [12.8\%] vs 6 [1.1\%], $p$-value $<0.001)$, respectively. In contrast, anosmia/dysgeusia was more prevalent in non-hospitalised children (73 [13.3\%] vs 3 [2.4\%], $p$-value 0.001$)$. Children admitted to ICU more often had abdominal pain (5 [33.3\%] vs 11 [10.0\%], $p$-value 0.034$)$ and rash (5 [33.3\%] vs 11 [10.0\%], $p$-value 0.034$)$ than non-ICU-hospitalised children. A heatmap with a co-occurrence matrix for symptoms showed three clusters of symptoms representing three different clinical phenotypes (Fig. 3). The first cluster represents an upper respiratory tract illness with fever, cough, rhinorrhoea and pharyngitis; the second a gastrointestinal illness with abdominal pain, diarrhoea and vomiting, and the third cluster corresponds to more constitutional symptoms with headache, myalgia and asthenia.

\section{Complications}

A total of 28 (4.1\%) children with SARS-CoV-2 infection developed complications; this was more frequent in hospitalised 
Table 2 Comorbidities in children with SARS-CoV-2 infection. Note that some patients had more than one comorbidity reported

\begin{tabular}{|c|c|c|c|c|}
\hline \multirow[b]{3}{*}{ Any pre-existing comorbidities } & \multirow{3}{*}{$\begin{array}{l}\text { Overall } \\
n(\%) \\
106(100)\end{array}$} & \multirow{3}{*}{$\begin{array}{l}\text { Non- } \\
\text { hospitalised } \\
n(\%) \\
72(13)\end{array}$} & \multirow{3}{*}{$\begin{array}{l}\text { Hospitalised* } \\
\boldsymbol{n}(\%) \\
\mathbf{3 4}(\mathbf{2 7 . 0})\end{array}$} & \multirow{3}{*}{$\begin{array}{l}\text { ICU } \\
n(\%) \\
5(31.3)\end{array}$} \\
\hline & & & & \\
\hline & & & & \\
\hline Respiratory disease & $45(42.5)$ & $31(43.1)$ & $14(41.2)$ & $4(80.0)$ \\
\hline Asthma/bronchitis & 38 & 30 & 8 & 3 \\
\hline $\begin{array}{l}\text { Obstructive sleep apnoea/adenoid } \\
\text { hypertrophy }\end{array}$ & 2 & 0 & 2 & 0 \\
\hline Cystic fibrosis/primary ciliary dyskinesia & 2 & 0 & 2 & 0 \\
\hline Apnoea of prematurity & 2 & 0 & 2 & 1 \\
\hline Bronchopulmonary dysplasia & 1 & 1 & 0 & 0 \\
\hline Endocrinological disease & $15(14.2)$ & $12(16.7)$ & 4 (11.8) & $\mathbf{0}$ \\
\hline Obesity & 10 & 8 & 2 & 0 \\
\hline Diabetes mellitus type 1 & 5 & 3 & 2 & 0 \\
\hline Hashimoto thyroiditis & 1 & 1 & 0 & 0 \\
\hline Haemato-oncological disease & $12(11.3)$ & $5(6.9)$ & 7 (20.6) & $1(20.0)$ \\
\hline Leukaemia & 5 & 2 & 3 & 0 \\
\hline Thalassemia/sickle-cell disease & 2 & 1 & 1 & 0 \\
\hline Neutropenia & 2 & 0 & 2 & 1 \\
\hline Medulloblastoma & 1 & 1 & 0 & 0 \\
\hline Severe anaemia & 1 & 0 & 1 & 0 \\
\hline Glucose-6-dehydrogenase deficiency & 1 & 1 & 0 & 0 \\
\hline Cardiovascular disease & $10(9.4)$ & $5(6.9)$ & $5(14.7)$ & $\mathbf{0}$ \\
\hline Congenital heart defect & 6 & 2 & 4 & 0 \\
\hline Post heart transplant & 1 & 0 & 1 & 0 \\
\hline Myocarditis & 1 & 1 & 0 & 0 \\
\hline Hypertrophic cardiomyopathy & 1 & 1 & 0 & 0 \\
\hline Familial long QT syndrome & 1 & 1 & 0 & 0 \\
\hline Neurologic disease & $8(7.5)$ & $4(5.6)$ & $4(11.8)$ & $1(20.0)$ \\
\hline Psychiatric & 2 & 0 & 2 & 0 \\
\hline Epilepsy & 2 & 2 & 0 & 0 \\
\hline Microcephaly & 1 & 0 & 1 & 1 \\
\hline Neurofibromatosis & 1 & 1 & 0 & 0 \\
\hline Multiple strokes & 1 & 0 & 1 & 0 \\
\hline Autistic spectrum disorder & 1 & 1 & 0 & 0 \\
\hline Surgical comorbidities & $6(5.7)$ & $5(6.9)$ & $1(2.9)$ & $\mathbf{0}$ \\
\hline Immunodeficiency & $5(4.7)$ & $2(2.8)$ & $3(8.8)$ & $\mathbf{0}$ \\
\hline Commune variable immune deficiency & 1 & 0 & 1 & 0 \\
\hline Microdeletion 22q11 & 1 & 0 & 1 & 0 \\
\hline Autoimmune lymphoproliferative syndrome & 1 & 1 & 0 & 0 \\
\hline Complement activation deficiency & 1 & 1 & 0 & 0 \\
\hline Status post renal transplant & 1 & 0 & 1 & 0 \\
\hline Nephrological disease & $5(4.7)$ & $1(1.4)$ & $4(11.8)$ & $\mathbf{0}$ \\
\hline Urolithiasis & 1 & 0 & 1 & 0 \\
\hline Hydronephrosis & 1 & 1 & 0 & 0 \\
\hline Kidney failure & 2 & 0 & 2 & 0 \\
\hline Autosomal recessive polycystic kidney disease & 1 & 0 & 1 & 0 \\
\hline Genetic disease & $4(3.8)$ & $2(2.8)$ & $2(5.9)$ & $\mathbf{0}$ \\
\hline Von Hippel Lindau & 1 & 1 & 0 & 0 \\
\hline Down disease & 2 & 1 & 1 & 0 \\
\hline Mowat Wilson syndrome & 1 & 0 & 1 & 0 \\
\hline
\end{tabular}


Table 2 (continued)

\begin{tabular}{lllll}
\hline & Overall & $\begin{array}{l}\text { Non- } \\
\text { hospitalised }\end{array}$ & Hospitalised* & ICU \\
Any pre-existing comorbidities & $\boldsymbol{n}(\%)$ & $\boldsymbol{n}(\%)$ & $\boldsymbol{n}(\%)$ & $\boldsymbol{n}(\%)$ \\
\hline Auto-immune disease & $\mathbf{1 0 6}(\mathbf{1 0 0})$ & $\mathbf{7 2}(\mathbf{1 3})$ & $\mathbf{3 4}(\mathbf{2 7 . 0 )}$ & $\mathbf{5}(\mathbf{3 1 . 3})$ \\
Juvenile idiopathic arthritis & $\mathbf{4 ( 3 . 8 )}$ & $\mathbf{3 ( 4 . 2 )}$ & $\mathbf{1 ( 2 . 9 )}$ & $\mathbf{0}$ \\
Granulomatosis with polyangiitis & 3 & 3 & 0 & 0 \\
Auto-inflammatory disease & 1 & 0 & 1 & 0 \\
Cryopyrin-associated periodic syndrome & 1 & 1 & $\mathbf{0}$ & $\mathbf{0}$ \\
Familial Mediterranean fever & 1 & 1 & 0 & 0 \\
Chronic multifocal osteomyelitis & 1 & 1 & 0 & 0 \\
Gastroenterological disease & $\mathbf{2 ( 1 . 9 )}$ & $\mathbf{2 ( 2 . 8 )}$ & $\mathbf{0}$ & 0 \\
Gastritis & 1 & 1 & 0 & $\mathbf{0}$ \\
Coeliac disease & 1 & 1 & 0 & 0 \\
Hepatological disease & $\mathbf{2 ( 1 . 9 )}$ & $\mathbf{2 ( 2 . 8 )}$ & $\mathbf{0}$ & 0 \\
Cholestasis & 2 & 2 & 0 & $\mathbf{0}$ \\
Prematurity & $\mathbf{2 ( 1 . 9 )}$ & $\mathbf{1}(\mathbf{1 . 4})$ & $\mathbf{1}(\mathbf{2 . 9})$ & 0 \\
\hline
\end{tabular}

*Numbers of hospitalised children include those admitted to intensive care unit (ICU)

than non-hospitalised children ( $p$-value $<0.001)$. The most frequent complications/non-pulmonary organ manifestations were cardiovascular in $12(1.8 \%)$ children, including coronary artery dilatation $(n=4)$, elevation of cardiac enzymes $(n=3)$, hypotensive shock $(n=3)$, myocarditis $(n=1)$, vasculitis $(n=3)$ and thromboembolism $(n=1)$. Bacterial co-infection was reported in $8(1.2 \%)$ children, exclusively in hospitalised children. Further complications were pancytopenia $(n=5)$, kidney failure $(n=4)$, seizures $(n=3)$, encephalopathy $(n=1)$, polyradiculoneuritis $(n=1)$ and myopathy $(n=1)$. No nosocomial infections were reported.

In hospitalised children, three $(2.4 \%)$ deaths were reported during the study period. A 10-month-old infant with severe brain oedema, signs of hypoxic-ischemic encephalopathy and cerebral haemorrhages presenting with vomiting without fever, respiratory distress (with evidence of respiratory illness on chest radiography) and status epilepticus. A 2-month-old infant with cardiorespiratory arrest secondary to cerebral haemorrhage presenting with acholic stools, haematochezia, haematemesis and hepatosplenomegaly with abnormal liver function. A 6-year-old with an Epstein-Barr Virus (EBV)-associated hemophagocytic lymphohistiocytosis developed cerebral haemorrhage due to coagulation deficiency caused by liver failure. He presented with abdominal pain, fever and abnormal liver function 2 weeks after a SARS-CoV-2 infection and was initially suspected to have PIMS-TS.

\section{Diagnosis}

Diagnosis of SARS-CoV-2 infection was mostly confirmed by PCR (663 [98.1\%]). Of the 40 children in
Table 3 Univariable and multivariable regression analysis for risk of hospitalisation. Note, the number of specific comorbidities was too low to be included in the multivariable analysis

\begin{tabular}{|c|c|c|c|c|c|c|}
\hline & \multicolumn{3}{|c|}{ Univariable regression } & \multicolumn{3}{|c|}{ Multivariable regression } \\
\hline & OR & 95\% CI & $p$-value & OR & 95\% CI & p-value \\
\hline $\begin{array}{l}\text { Age }<2 \text { years } \\
\text { (Reference) }\end{array}$ & 1.23 & 0.85 to 1.78 & 0.27 & 1.52 & 0.72 to 3.24 & 0.27 \\
\hline Age 2 to $<5$ years & 0.14 & 0.05 to 0.31 & $<0.01$ & 0.10 & 0.04 to 0.24 & $<0.01$ \\
\hline Age 5 to $<10$ years & 0.13 & 0.07 to 0.26 & $<0.01$ & 0.11 & 0.05 to 0.22 & $<0.01$ \\
\hline Age $>10$ years & 0.09 & 0.05 to 0.14 & $<0.01$ & 0.08 & 0.05 to 0.13 & $<0.01$ \\
\hline Gender (male) & 0.81 & 0.55 to 1.20 & 0.31 & 0.78 & 0.50 to 1.21 & 0.27 \\
\hline Comorbidities (Any) & 2.49 & 1.55 to 3.95 & $<0.01$ & 3.23 & 1.89 to 5.50 & $<0.01$ \\
\hline Cardiac comorbidities & 4.56 & 1.25 to 16.63 & 0.02 & - & - & - \\
\hline Respiratory comorbidities & 2.12 & 1.06 to 4.04 & 0.03 & - & - & - \\
\hline Immunodeficiency & 6.76 & 1.11 to 51.75 & 0.04 & - & - & - \\
\hline Haemato-oncological comorbidities & 6.49 & 2.04 to 22.26 & $<0.01$ & - & - & - \\
\hline
\end{tabular}




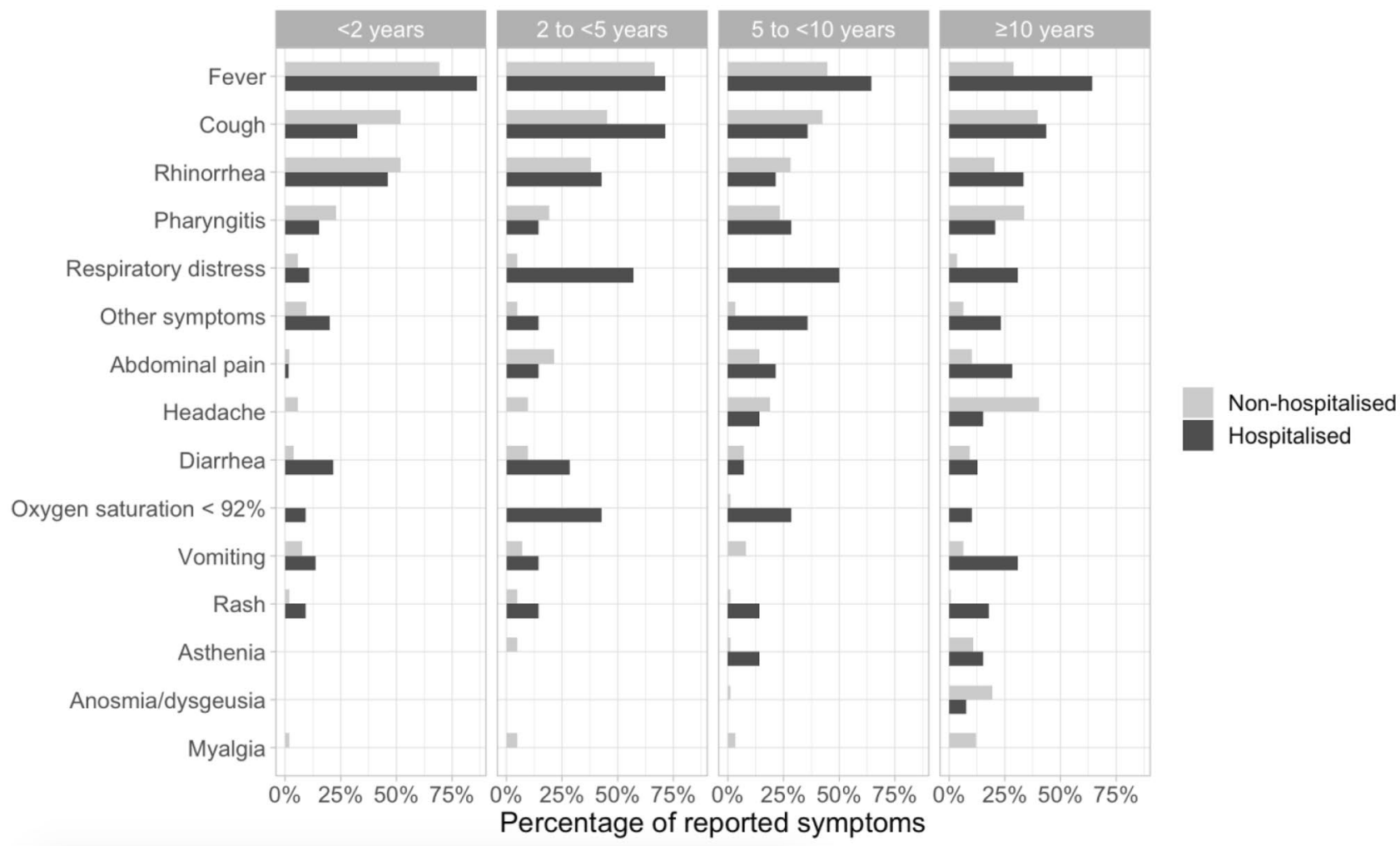

Fig. 2 Symptoms distribution in non-hospitalised and hospitalised children with SARS-CoV-2 infections according to age group. Other symptoms included conjunctivitis, otalgia, cheilitis, hand oedema, thoracic pain, arthralgia, acrocyanosis, fainting, seizure, alguria, orchitis and macro-haematuria

whom serology was done, 35 (87.5\%) were positive. Five ICU-admitted patients had positive serology with negative PCR. A chest radiography was done in 47 (6.9\%) children and showed unilateral and bilateral changes in six (12.8\%) and $16(34.0 \%)$ cases, respectively. Echocardiography was done in $47(6.9 \%)$ children; abnormal findings were identified in eight $(21.6 \%)$ children (coronary dilatation $(n=4)$, reduced left ventricle ejection fraction $(n=3)$ and dyskinesia $(n=1))$, and all of them were hospitalised. Other diagnostic tests included abdominal ultrasound (16 [2.4\%]) and thoracic computed tomography (9 [1.3\%]) (further details in supplementary data Table S1).

\section{Transmission}

In total, 309 children (45.6\%) had a family member with a confirmed or suspected SARS-CoV-2 infection (supplementary data Fig. S4). Community-acquired infection (including school and day-care) was confirmed or suspected in 86 $(12.7 \%)$ children. In one-third of children, the primary case was unknown $284(41.9 \%)$.

\section{Discussion}

This nationwide study presents the first epidemiological data from active surveillance of SARS-CoV-2 infection in non-hospitalized and hospitalized children in Switzerland. Data have been collected during both pandemic waves: the first wave was marked by general lockdown with school closures. The second wave was characeristed by an important rise of cases in October, which was similar to the evolution of the pandemic in the adult population and was followed by a second lockdown in November [17]. Our findings suggest that comorbidities are an important factor associated with two times higher hospitalisation rates in children and adolescents. Data from the Centres of Disease Control surveillance of in the USA suggest that overall hospitalisation rates are six times higher among children with a reported underlying condition [18]. In children $\leq 9$ years of age, the rate of underlying conditions was reported to be $4.1 \%$ overall and $22.3 \%$ in those hospitalised. We have found similar rates of comorbidities in hospitalised but higher rates in non-hospitalised children. The latter may be explained by the fact that our data are based on a hospital-centred surveillance, which 


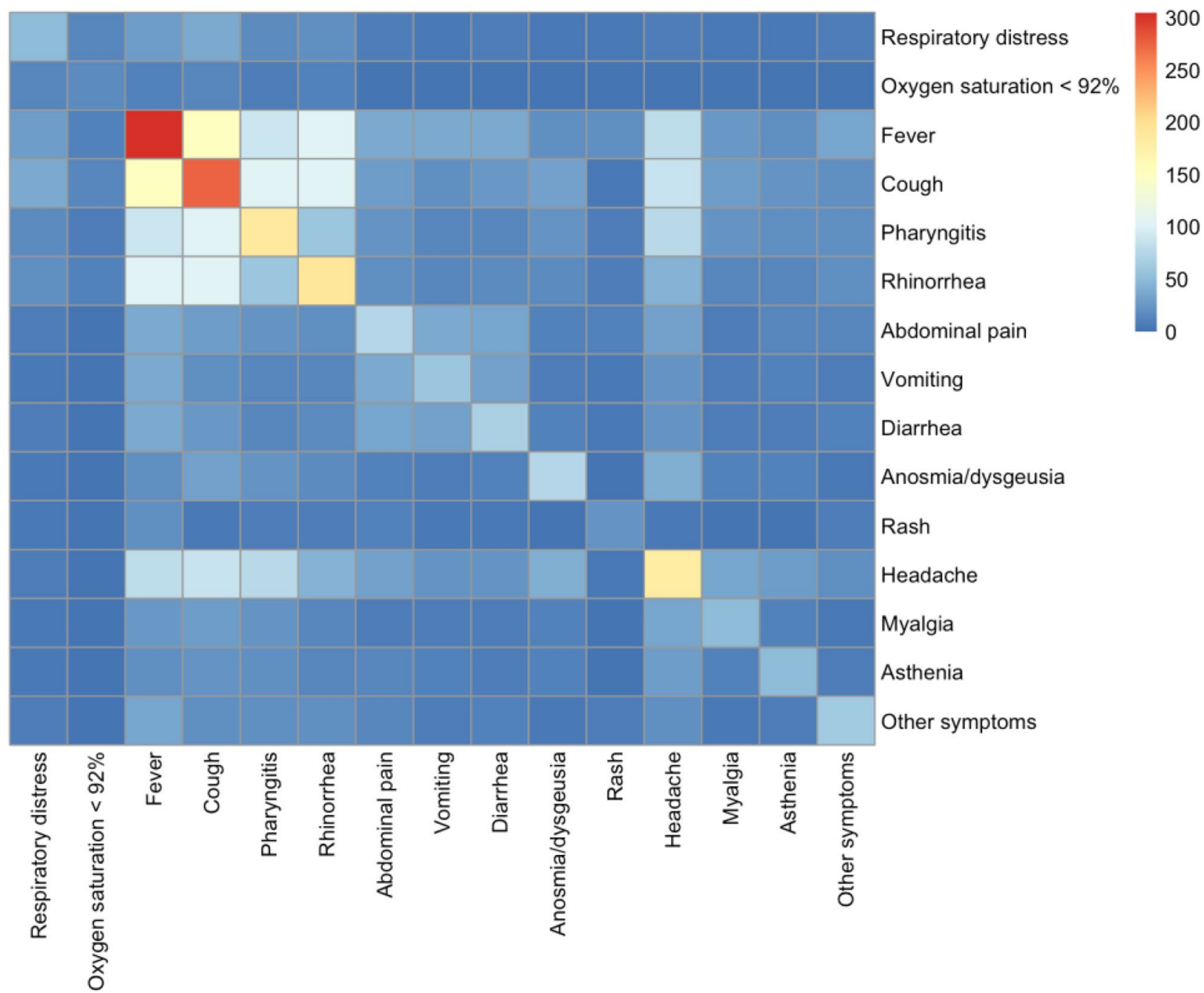

Fig. 3 Heatmap using k-means with symptom cluster (the colour code stands for the number of children with corresponding symptoms)

likely underestimates the burden of disease in non-hospitalised children. In addition, children with comorbidities were more likely tested and referred to hospitals, particularly in the early phase of the pandemic. This is also reflected by the fact that we observed a more severe spectrum of paediatric COVID-19 with a lower rate of asymptomatic children compared to other studies $[19,20]$. Another paediatric study from the UK reported much higher rates of comorbidities of $42 \%$ in hospitalised children, but this study did not include data on non-hospitalised cases [1]. The most frequent comorbidities reported in the UK study were neurological, asthma and haemato-oncological or immunological which compares to the spectrum reported in our study. Whether underlying conditions are associated with increased severity of disease or a lower threshold for admission remains unclear based on our data.
In our setting, $13 \%$ of hospitalised children required admission to ICU, which is in line with other European studies [1, 19,21]. Similarly, to other studies, children with serious medical conditions (immunodeficiency, haematooncological, cardiac or metabolic disease) in our cohort did not develop severe COVID-19 requiring ICU admission more often than previously healthy children [22]. A recent systematic review suggests that children with underlying cardiac disease might be at increased risk of severe COVID-19 [23]. However, we did not find this in our study population, where children with underlying cardiac disease (including one patient after heart transplantation) did not experience a severe disease course. Three children with asthma/bronchitis required ICU admission due to respiratory failure. Evidence are sparse on whether asthma is a risk factor for severe COVID-19 in children; a systematic review found only two 
reports considering asthma as a risk factor for SARS-CoV-2 infection [24]. However, a large US retrospective paediatric study found that the risk of testing positive for SARS$\mathrm{CoV}-2$ was reduced in children with a respiratory disorder, including asthma [25]. Moreover, children with asthma are underrepresented during this pandemic, and studies in adults have not identified SARS-CoV-2 as a trigger for asthma exacerbations [26]. Several hypotheses have been proposed to explain this unexpected finding, including an impaired immune response to SARS-CoV-2. Children with asthma have lower levels of interferon-gamma and consequently reduced angiotensin-converting enzyme 2 (ACE-2) gene expression in airway epithelium, which act as the entry point of SARS-CoV-2 into the human body [26]. Other studies report reduced ACE-2 expression in patients with allergic asthma or after inhaled corticosteroids [27, 28].

Our study clearly shows that the clinical spectrum of COVID-19 is different in hospitalised and non-hospitalised children with fever and rash being more common in those admitted, but cough, rhinorrhoea and pharyngitis being comparable. Although fever was the most frequent symptom, especially among young children, the incidence was lower than reported by other multicentric studies [1, 19, 21]. This lower prevalence might reflect the higher proportion of adolescents in our cohort. Age is a further important factor influencing the clinical spectrum of disease with fever and respiratory symptoms of lower severity being more frequent in children below 2 years of age. Older children more likely present with non-respiratory symptoms, and anosmia/ dysgeusia was only rarely recorded in children less than 10 years of age. A similar spectrum was described in the UK paediatric cohort; however, this only included hospitalised children [1].

Our findings confirm that paediatric COVID-19 is mostly a mild illness. Several hypothesis have been proposed to explain the milder disease seen in children, including an age-related difference in the immune response, with a stronger innate immune response in children and agerelated differences in expression of ACE-2 [5, 29]. Our data also captures cases with the suspicion of PIMS-TS corresponding to the most severe spectrum of COVID-19. Of the three deaths reported, two were being temporally related to SARS-CoV-2 infection without another disease identified.

Evidence-based treatment options for paediatric COVID-19 are still lacking [30]. In our study, the majority of non-hospitalised but also hospitalised children did not receive specific treatment. However, children admitted to ICU with PIMS-TS required inotropic support and received systemic corticosteroid and IVIG in combination with immunomodulators (including anakinra (interleukin-1 inhibitor) and tocilizumab (interleukin-6 inhibitor)). Similarly, most case series report using IVIG and/ or corticosteroid as main therapeutic option for children with PIMS-TS features, given the similitude with Kawasaki disease [14, 15, 31-34]. The rational for using anticytokine agents (anakinra or tocilizumab) is that these toxic shock-like clinical presentations are caused by exaggerated immune response and excessive cytokine release, called cytokine storm, rather than by viral replication itself [35]. However, there are insufficient data to recommend for or against the use of specific treatment for PIMS-TS, and management remains centred on effective supportive care [30].

The strengths of our study are the multicentre nationwide study design capturing the widespread epidemiology of SARS-CoV-2 infection in a European country, the inclusion of a large number of children including non-hospitalised children and the detailed demographic and clinical data. However, our study overestimates the admission rate as we did not include patients tested in private practices and as some children were hospitalised for reasons other than SARS-CoV-2 infection. Furthermore, the threshold for admission might have been different depending on local guidelines, pre-existing comorbidities, physicians' discretion and presentations outside office hours. Data from the Federal Office of Public Health show a $0.9 \%$ admission rate in children in the same time period (personal communication from Mirjam Mäusezahl, Federal Office of Public Health, 23 March 2021). In addition, in the early weeks of the COVID-19 pandemic, SARS-CoV-2 testing was not considered a priority in children, and only children with severe symptoms or persistent fever were tested. Testing strategies also differed regionally which may have affected some of the observed regional differences. Analysis of weight and obesity as a potential risk factor for hospitalisation could not be included as weight was not recorded in most non-hospitalised cases. We are also unable to analyse children classified as PIMS-TS in detail as specific data on PIMS-TS cases are only prospectively collected since November 2020. Due to the important rise of cases in October 2020, data collection of SPSU SARS-CoV-2 for non-hospitalised children was stopped, and therefore, we are unable to include nonhospitalised children in the data analysis during the further second wave.

\section{Conclusion}

This study confirms that COVID-19 is mostly a mild disease in children and adolescents with low mortality. Fever, rash and comorbidities are associated with higher admission rates. The clinical spectrum and severity are influenced by age in paediatric COVID-19. Continuous observation is necessary to further understand paediatric COVID-19, guide therapy and evaluate the necessity for vaccination in children. 
Supplementary Information The online version contains supplementary material available at https://doi.org/10.1007/s00431-021-04276-9.

Acknowledgements We thank the representatives of the paediatric units in Switerzland: M. Albisetti; V. Bernet; C. Betti; F. Cachat; P. Caplazi; M-L. Decker; E. Durrer; S. Fluri; M. Gebauer; M. Gehri; E. Giannoni; S. Grupe; M. Horn; A. L'Huiller; T. Karen; E. Kellner; G. Laube; B. Laubscher; J. Llor; F. Luterbacher; H. Madlon; A. Malzacher; M. Martins; J. McDougall; A. Merglen; S. Minocchieri; V. Muehlethaler; T. Neuhaus; A. Niederer; S. Nikorelou; M. Plebani; Ratnasabapathy; C. Relly, T. Riedel; M. Russo; H. Schmid; K. Staudacher; M. Torres Escobar; J. Wildhaber; A. Wörner; A. Zemmouri. We would also like to acknowledge the administrative support by Daniela Beeli, Mirjam Mäusezahl and Fabian Tschagellar, from the Federal Office of Public Health Switzerland.

Authors' contributions This study was designed by PZ and NR. Data analysis was done by AU, PZ and NR. AU wrote the first draft; all the authors revised the manuscript and approved the final draft.

Funding Open access funding provided by University of Basel. The study is supported by the Swiss Federal Office of Public Health and has received grants from the Swiss Society of Paediatrics and the Paediatric Infectious Disease Group of Switzerland.

Availability of data and material Data collected for the study and the study protocol will be made available to others on request.

\section{Declarations}

Ethics approval The study has received ethical approval by the Ethikkommission Nordwest- und Zentralschweiz (EKNZ 2020-01130).

\section{Consent to participate N/A}

\section{Consent for publication N/A}

Competing interests The authors declare no competing interests.

Open Access This article is licensed under a Creative Commons Attribution 4.0 International License, which permits use, sharing, adaptation, distribution and reproduction in any medium or format, as long as you give appropriate credit to the original author(s) and the source, provide a link to the Creative Commons licence, and indicate if changes were made. The images or other third party material in this article are included in the article's Creative Commons licence, unless indicated otherwise in a credit line to the material. If material is not included in the article's Creative Commons licence and your intended use is not permitted by statutory regulation or exceeds the permitted use, you will need to obtain permission directly from the copyright holder. To view a copy of this licence, visit http://creativecommons.org/licenses/by/4.0/.

\section{References}

1. Swann OV et al (2020) Clinical characteristics of children and young people admitted to hospital with covid-19 in United Kingdom: Prospective multicentre observational cohort study. BMJ 370:m3249
2. Ludvigsson JF (2020) Systematic review of COVID-19 in children shows milder cases and a better prognosis than adults. 1088 I Acta Paediatr 109:1088-1095

3. Zimmermann P, Curtis N (2020) Coronavirus infections in children including COVID-19. Pediatr Infect Dis J 39:355-368

4. Dong Y et al (2020) Epidemiology of COVID-19 among children in China. Pediatrics 145:e20200702

5. Zimmermann P, Curtis N (2020) Why is COVID-19 less severe in children ? A review of the proposed mechanisms underlying the age-related difference in severity of SARS-CoV-2 infections. 1-11. https://doi.org/10.1136/archdischild-2020-320338

6. Oberweis ML et al (2020) Pediatric life-threatening coronavirus disease 2019 with myocarditis. Pediatr Infect Dis J Publish Ah 1088-1095

7. Riphagen S, Gomez X, Gonzalez-martinez C, Wilkinson N, Theocharis P (2020) Correspondence hyperinflammatory shock in children during. Lancet 6736:2019-2020

8. Zimmermann P, Goetzinger F, Ritz N (2020) Additional concerns regarding children with coronavirus disease 2019. JAMA Pediatr 174:1216

9. Verdoni L et al (2020) An outbreak of severe Kawasaki-like disease at the Italian epicentre of the SARS-CoV-2 epidemic: an observational cohort study. Lancet 395:1771-1778

10. Viner RM, Whittaker E (2020) Kawasaki-like disease: emerging complication during the COVID-19 pandemic. Lancet 395:1741-1743

11. Jones VG et al (2020) COVID-19 and Kawasaki disease: novel virus and novel case. https://doi.org/10.1542/hpeds.2020-0123

12. Toubiana $\mathbf{J}$ et al (2020). Kawasaki-like multisystem inflammatory syndrome in children during the covid-19 pandemic in Paris, France: prospective observational study. BMJ. Jun 3;369:m2094. https://doi. org/10.1136/bmj.m2094

13. Levin M (2020). E d i t o r i a 1 Childhood multisystem inflammatory syndrome-a new challenge in the pandemic. https://doi.org/ 10.1056/NEJMoa2021756

14. Moraleda C et al (2020) Multi-inflammatory syndrome in children related to severe acute respiratory syndrome coronavirus 2 (SARSCoV-2) in Spain. Clin Infect Dis. https://doi.org/10.1093/cid/ciaa1042

15. Cheung EW et al (2020) Multisystem inflammatory syndrome related to COVID-19 in previously healthy children and adolescents in New York City. JAMA 324:294

16. Wright A (2016) REDCap: a tool for the electronic capture of research data. J Electron Resour Med Libr 13:197-201

17. Covid-19 Statistics Switzerland. Available at: https://www.covid19data.ch/. (Accessed: 2nd July 2021)

18. Stokes EK et al (2020) Coronavirus disease 2019 case surveillance - United States, January 22-May 30, 2020. MMWR Morb Mortal Wkly Rep 69:759-765

19. Parri $\mathrm{N}$ et al (2020) Characteristic of COVID-19 infection in pediatric patients: early findings from two Italian Pediatric Research Networks. Eur J Pediatr 179:1315-1323

20. Maltezou HC et al (2020) Children and adolescents with SARSCoV-2 infection: epidemiology, clinical course and viral loads. Pediatr Infect Dis J 39:e388-e392

21. Götzinger F et al (2020) COVID-19 in children and adolescents in Europe: a multinational, multicentre cohort study. Lancet Child Adolesc Heal 4:653-661

22. Issitt $\mathrm{R}$ et al (2020) Coronavirus (COVID-19) infection in children at a specialist centre: outcome and implications of underlying high-risk comorbidities in a paediatric population. https://doi.org/ $10.1101 / 2020.05 .20 .20107904$

23. Williams $\mathrm{N}$ et al (2020) COVID-19 Severe acute respiratory syndrome coronavirus 2 (SARS-CoV-2) infection in children and adolescents: a systematic review of critically unwell children and the association with underlying comorbidities. Eur J Pediatr 2 
24. Castro-Rodriguez JA, Forno E (2020) Asthma and COVID-19 in children: a systematic review and call for data. Pediatr Pulmonol 55:2412-2418

25. Bailey LC et al (2020) Assessment of 135794 pediatric patients tested for severe acute respiratory syndrome coronavirus 2 across the United States. JAMA Pediatr 1-9. https://doi.org/10.1001/ jamapediatrics.2020.5052

26. Wang J, Pawankar R, Tsai H, Wu LS, Kuo W (2020) COVID-19 and asthma, the good or the bad? Allergy all.14480. https://doi. org/10.1111/all.14480

27. Jackson DJ et al (2020) Association of respiratory allergy, asthma, and expression of the SARS-CoV-2 receptor ACE2. J Allergy Clin Immunol 146:203-206.e3

28. Peters MC et al (2020) COVID-19-related genes in sputum cells in asthma: relationship to demographic features and corticosteroids. Am J Respir Crit Care Med 202:83-90

29. Bunyavanich S, Do A, Vicencio A (2020) Nasal gene expression of angiotensin-converting enzyme 2 in children and adults. JAMA $323: 2427$

30. National Institute of Health (2020) COVID-19 Treatment Guidelines Panel. Coronavirus disease 2019 (COVID-19) treatment guidelines. National Institutes of Health. NIH 2019:130
31. Tanner T, Wahezi DM (2020) Hyperinflammation and the utility of immunomodulatory medications in children with COVID-19. Paediatr Respir Rev 35:81-87

32. Whittaker E et al (2020) Clinical Characteristics of 58 children with a pediatric inflammatory multisystem syndrome temporally associated with SARS-CoV-2 group information: the PIMS-TS Study Group and EUCLIDS and PERFORM Consortia members are listed in Supplement 1. JAMA 324:259-269

33. Viner RM et al (2020) Susceptibility to SARS-CoV-2 infection among children and adolescents compared with adults a systematic review and meta-analysis Editorial Supplemental content. https://doi.org/10.1001/jamapediatrics.2020.4573

34. Verdoni L et al (2020) An outbreak of severe Kawasaki-like disease at the Italian epicentre of the SARS-CoV-2 epidemic: an observational cohort study. www.thelancet.com 395

35. Sanders JM, Monogue ML, Jodlowski TZ, Cutrell JB (2020) Pharmacologic treatments for coronavirus disease 2019 (COVID-19): a review. JAMA - J Am Med Assoc 323:1824-1836

Publisher's Note Springer Nature remains neutral with regard to jurisdictional claims in published maps and institutional affiliations.

\title{
Authors and Affiliations
}

\author{
Anita Uka ${ }^{1,2} \cdot$ Michael Buettcher $^{3} \cdot$ Sara Bernhard-Stirnemann ${ }^{4} \cdot$ Yves Fougère $^{5} \cdot$ Dehlia Moussaoui $^{6}$. \\ Lisa Kottanattu ${ }^{7}$. Noémie Wagner ${ }^{6} \cdot$ Petra Zimmermann $^{1,2,8} \cdot$ Nicole Ritz $^{9,10,11}$. Swiss Paediatric Surveillance Unit \\ (SPSU) \\ Anita Uka \\ anita.uka@unifr.ch \\ Michael Buettcher \\ michael.buettcher@luks.ch \\ Sara Bernhard-Stirnemann \\ sara.bernhard@ksa.ch \\ Yves Fougère \\ yves.fougere@chuv.ch \\ Dehlia Moussaoui \\ Dehlia.Moussaoui@hcuge.ch \\ Lisa Kottanattu \\ Lisa.Kottanattu@eoc.ch \\ Noémie Wagner \\ Noemie.Wagner@hcuge.ch \\ Petra Zimmermann \\ petra.zimmermann@unifr.ch \\ Swiss Paediatric Surveillance Unit (SPSU) \\ spsu@bag.admin.ch \\ 1 Faculty of Science and Medicine, University of Fribourg, \\ Fribourg, Switzerland \\ 3 Paediatric Infectious Diseases, Children's Hospital Lucerne, \\ Luzern, Switzerland \\ 4 Department of Paediatrics, Children's Hospital, Cantonal \\ Hospital Aarau, Aarau, Switzerland \\ 5 Pediatric Infectious Diseases and Vaccinology Unit, \\ Department Women-Mother-Child, Lausanne University \\ Hospital, Lausanne, Switzerland \\ 6 Department of Paediatrics, Gynaecology and Obstetrics, \\ General Paediatrics Division, Geneva University Hospitals, \\ Geneva, Switzerland \\ 7 Ente Ospedaliero Cantonale, Istituto Pediatrico Della \\ Svizzera Italiana, Bellinzona, Switzerland \\ 8 Infectious Diseases Research Group, Murdoch Children's \\ Research Institute, Parkville, Australia \\ $9 \quad$ Paediatric Infectious Diseases and Vaccinology, University \\ of Basel Children's Hospital, Basel, Switzerland \\ 10 Department of Paediatrics, The Royal Children's Hospital \\ Melbourne, The University of Melbourne, Melbourne, \\ Australia \\ 11 University Children's Hospital Basel UKBB, Spitalstrasse \\ 33, CH-4031 Basel, Switzerland
}

2 Department of Paediatrics, Fribourg Hospital HFR, Fribourg, Switzerland 